\title{
Interpolating Sequences for Bounded Harmonic Functions
}

\section{JOHN GARNETT}

\author{
Communicated by P. R. Halmos
}

Introduction. A sequence $\left\{z_{i}\right\}_{j=1}^{\infty}$ in the open unit disc $\triangle$ is an interpolating sequence if for every bounded sequence $\left\{a_{j}\right\}_{j=1}^{\infty}$ there is a bounded analytic function $f$ on $\triangle$ such that $f\left(z_{j}\right)=a_{j}$ for all $j$. If for every such $\left\{a_{j}\right\}_{j=1}^{\infty}$ there is a bounded harmonic function $u$ on $\triangle$ with $u\left(z_{i}\right)=a_{j}$, we call $\left\{z_{j}\right\}_{j=1}^{\infty}$ a harmonic interpolating sequence. It is clear that every interpolating sequence is a harmonic interpolating sequence. Our purpose here is to prove the converse.

Theorem. Every harmonic interpolating sequence is an interpolating sequence.

The proof uses Hall's lemma and the construction from the proof of the corona theorem given in [2]. The theorem given here had also been proved by Carleson by similar means (unpublished).

§1. Two elementary lemmas. Fix a harmonic interpolating sequence $\left\{z_{i}\right\}_{i=1}^{\infty}$.

Lemma 1. There are constants $M>1$ and $\eta=\eta(M)>0$ such that

$$
\left|\frac{z_{j}-z_{k}}{1-\bar{z}_{k} z_{j}}\right| \geqq \eta \quad \text { if } j \neq k
$$

(2) If $\left|a_{j}\right| \leqq 1$ for all $j$, there is $u$ harmonic with $u\left(z_{j}\right)=a_{i}$ and $\|u\|=$ $\sup |u(z)| \leqq M$.

Proof. The open mapping theorem for Banach spaces gives (2), and (1) is an easy consequence of (2). Indeed, let $u$ be real and harmonic with $u\left(z_{k}\right)=0$, $u\left(z_{j}\right)=1,\|u\| \leqq M$. If $v$ is the conjugate harmonic function vanishing at $z_{k}$, then $f(z)=e^{u+i v}-1$ satisfies $f\left(z_{k}\right)=0,\left|f\left(z_{j}\right)\right| \geqq e-1$, and $\|f\| \leqq e^{M}+1$. By Schwarz's lemma,

$$
\left|\frac{z_{i}-z_{k}}{1-\bar{z}_{k} z_{j}}\right| \geqq \frac{\left|f\left(z_{j}\right)\right|}{e^{M}+1} \geqq \frac{e-1}{e^{M}+1} .
$$

For any sequence $\left\{z_{j}\right\}_{j=1}^{\infty}$ in $\triangle$, let $\mu$ be the measure with mass $1-\left|z_{j}\right|^{2}$ at $z_{i}$. Function theoretic properties of $\left\{z_{i}\right\}_{i=1}^{\infty}$ can often be expressed in terms of $\mu$. 
For example, $\mu$ is finite if and only if $\left\{z_{i}\right\}_{i=1}^{\infty}$ is a Blaschke sequence [4]. Similarly we have

Lemma 2. Let $\left\{z_{i}\right\}_{j=1}^{\infty}$ be a sequence in $\triangle$ satisfying (1). Then $\left\{z_{j}\right\}_{i=1}^{\infty}$ is an interpolating sequence if and only if there is a constant $A$ such that for every set of the form $S=\left\{r>1-\delta,\left|\theta-\theta_{0}\right|<\delta\right\}$,

$$
\mu(S) \leqq A \cdot \delta .
$$

Proof. By a theorem of Carleson [2], [3], related to the Hardy-Littlewood maximal theorem, condition (3) implies there is a constant $c_{1}$ such that

$$
\sum_{k}\left(1-\left|z_{k}\right|^{2}\right)\left|g\left(z_{k}\right)\right|=\int|g(z)| d \mu \leqq \frac{c_{1}}{2 \pi} \int_{0}^{2 \pi}\left|g\left(e^{i \theta}\right)\right| d \theta
$$

for all $g \varepsilon H^{1}$. Taking $g(z)=\left(1-\left|z_{j}\right|^{2}\right) /\left(1-\bar{z}_{j} z\right)^{2}$ in (4), we have

$$
\sum_{k} \frac{\left(1-\left|z_{k}\right|^{2}\right)\left(1-\left|z_{j}\right|^{2}\right)}{\left|1-\bar{z}_{j} z_{k}\right|^{2}} \leqq c_{1} .
$$

Since

$$
1-\left|\frac{z_{j}-z_{k}}{1-\bar{z}_{j} z_{k}}\right|^{2}=\frac{\left(1-\left|z_{k}\right|^{2}\right)\left(1-\left|z_{i}\right|^{2}\right)}{\left|1-\bar{z}_{j} z_{k}\right|^{2}},
$$

(1) and (5) yield

$$
\log \prod_{k, k \neq j}\left|\frac{z_{i}-z_{k}}{1-\bar{z}_{k} z_{j}}\right|^{-2} \leqq c_{2} \sum_{k, k \neq j} 1-\left|\frac{z_{k}-z_{i}}{1-\bar{z}_{k} z_{i}}\right|^{2} \leqq c_{1} c_{2},
$$

so that for all $j$,

$$
\prod_{k, k=j}\left|\frac{z_{j}-z_{k}}{1-\bar{z}_{k} z_{j}}\right| \geqq \gamma>0 .
$$

Now (6) is a necessary and sufficient condition for interpolation ([1], [3], or [4]). On the other hand, it is well known that any interpolation sequence satisfies (4) (see for example p. 72 of [5]), and (4) is equivalent to (3) ([2], [3]).

We will prove the theorem by verifying (3) directly.

§2. Proof of the Theorem. We return to our fixed harmonic interpolating sequence $\left\{z_{j}\right\}_{j=1}^{\infty}$, and verify condition (3). By Lemma 2 that will prove the theorem. Fix $S=\left\{r>1-\delta,\left|\theta-\theta_{0}\right|<\delta\right\}$ and map $\triangle$ to the half plane $H=$ $\{y>0\}$ so that $S$ falls inside the square $Q=\{0<y<\delta, 0<x<\delta\}$ but inside no smaller such square. Retain the notation $\left\{z_{i}\right\}_{j=1}^{\infty}$ for the image sequence, but replace $\mu$ by the measure $\nu$ on $H$ with mass $\operatorname{Im} z_{j}$ at $z_{j}$. Condition (3) is equivalent to

$$
\nu(Q) \leqq A_{1} \cdot \delta .
$$

We will show $A_{1}$ depends only on $M$ and thus we suppose $\delta=1$.

Let $R^{n}$ denote the strip in $Q$ with $2^{-n-1}<y \leqq 2^{-n}$. By Lemma 1 and Harnack's 
theorem there is an integer $K=K(M)$ such that partitioning each $R^{n}$ into dyadic squares $S^{n}$ of side $2^{-n-K}$ we have:

(a) No $S^{n}$ contains more than one $z_{i}$.

(b) If $u$ is harmonic on $H$ and $\|u\| \leqq 2 M$, then the oscillation of $u$ over any $S^{n}$ is at most 1 .

For $z_{j} \varepsilon R^{n}$ let $I\left(z_{j}\right)$ be the horizontal segment of length $\left|I\left(z_{j}\right)\right|=2^{-K-n}$ through $z_{j}$ and inside some $S^{n}$. By (a) we have

$$
\nu(Q) \leqq 2^{K} \sum\left|I\left(z_{i}\right)\right| .
$$

Reindex the sum in (8) as follows: Say $z_{j}$ is in the first generation if no $I\left(z_{k}\right)$ lies above an interior point of $I\left(z_{j}\right)^{1}$.

Let $\left\{z_{p}\right\}$ denote the first generation. For each $z_{p}$ take a square $Q_{p}$ with base the projection $I\left(z_{p}\right)^{*}$ of $I\left(z_{p}\right)$ onto $\{y=0\}$. The $Q_{p}$ are shaded in the figure and their interiors are pairwise disjoint. Let $T_{p}$ be the rectangle between $I\left(z_{p}\right)$ and $Q_{p}$; it contains at most $A_{2}=A_{2}(M)$ intervals $I\left(z_{j}\right)$ by (a). So if $W^{0}=Q \backslash \cup Q_{p}$, then

$$
\nu\left(W^{0}\right)=\sum_{p} \nu\left(T_{p}\right) \leqq \sum_{p} A_{2}\left|I\left(z_{p}\right)\right| \leqq A_{2} .
$$

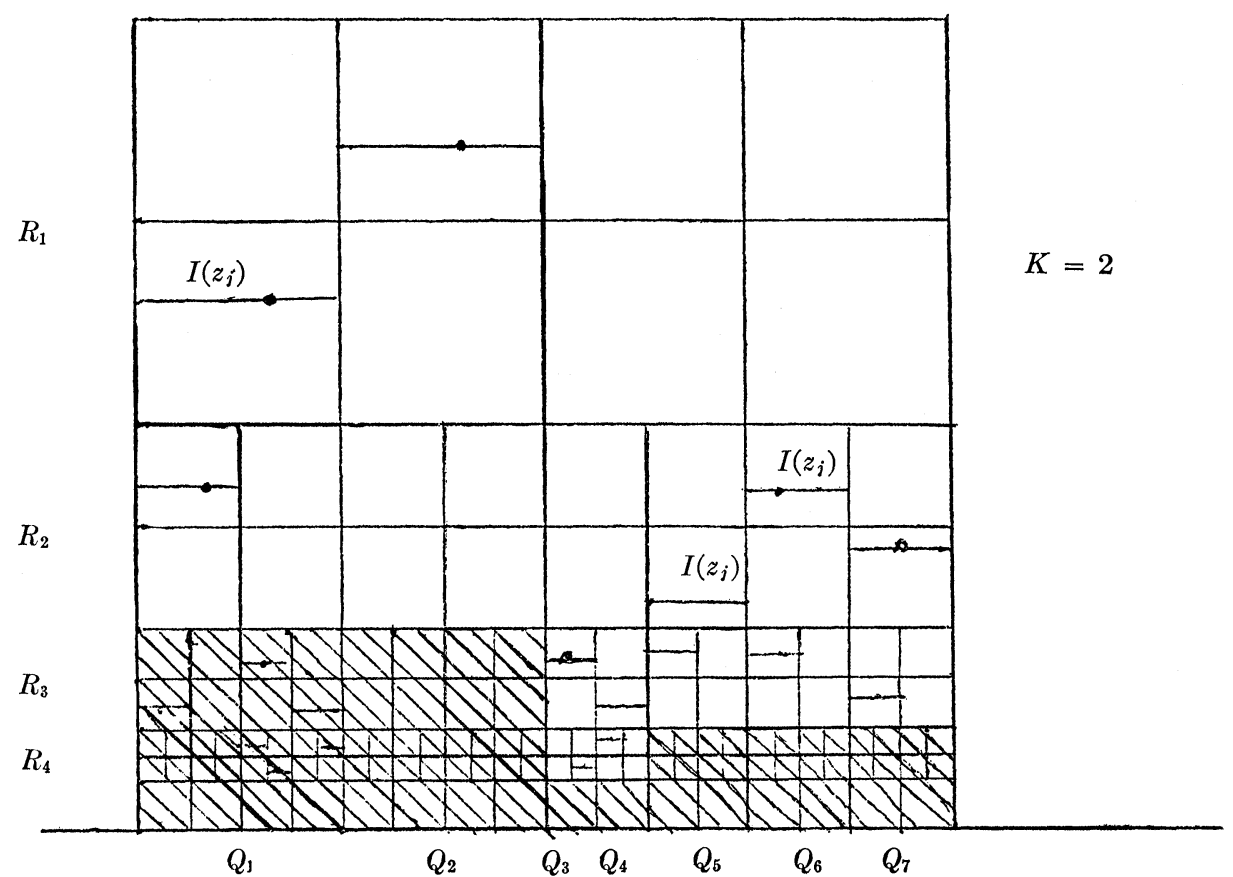

Repeat the above process inside each $Q_{p}$, obtaining a second generation $\left\{z_{p, q}\right\}$ of $z_{i}$ 's and new shaded squares $Q_{p, q} \subset Q_{p}{ }^{2}$.

1 If it is raining from the top of $Q, z_{j}$ is first generation precisely when $I\left(z_{j}\right)$ gets wet.

${ }^{2}$ Of course some $z_{j}$ in $T_{p}$ may fall in no generation, but then $\left|I\left(z_{j}\right)\right|$ has already been counted. 
Letting $W_{p}=Q_{p} \backslash \bigcup_{q} Q_{p, q}$ we have by (9) and a dilation, $\nu\left(W_{p}\right) \leqq A_{2}\left|I\left(z_{p}\right)\right|$. So if $W^{1}=\cup W_{p}$ and $E_{1}=\cup I\left(z_{p}\right)^{*}$ then

$$
\nu\left(W^{1}\right) \leqq A_{2}\left|E_{1}\right| \text {. }
$$

Now repeat the process with the $Q_{p, a}$ and with the corresponding squares for every later generation. We get sets $\left\{W^{k}\right\}_{k=0}^{\infty}$ and $\left\{E_{k}\right\}_{k=1}^{\infty}$ such that $E_{k+1} \subset$ $E_{k} \subset\{y=0\}$, and by (9) and (10)

$$
\nu(Q)=\sum_{k=0}^{\infty} \nu\left(W^{k}\right) \leqq A_{2}\left(1+\sum_{k=1}^{\infty}\left|E_{k}\right|\right) .
$$

Lemma 3. There exists $n=n(M)$ such that $\left|E_{k}\right| \leqq 1 / 2$ for $k \geqq n$.

Assuming the lemma for a moment, note that the $E_{k}$ are constructed iteratively so that then $\left|E_{k}\right|<2^{-m}$ if $k>n \cdot m$. Then by (11) we have

$$
\nu(Q) \leqq 2 n A_{2}
$$

which proves the theorem.

Proof of Lemma 3. Let $D$ be the strip $\{0<y<\pi\}$ and let $L_{k}=\bigcup\left\{I\left(z_{j}\right): z_{j}\right.$ is in the $k$-th generation $\}$. Thus its projection is $L_{k}^{*}=E_{k}$. Fix $n$, and set $G_{n}=$ $D \backslash \bigcup_{k=1}^{n} L_{k}, z^{*}=\pi i / 2 \varepsilon G_{n}$. Let $\lambda_{n}$ be the harmonic measure on $\partial G_{n}$ for $z^{*}$, let $a_{n}=\lambda_{n}\left(\bigcup_{k \leqq n} L_{k}\right)$, and reindexing the $L_{i}$ so that $\lambda_{n}\left(L_{n}\right)$ is largest, let $b_{n}=$ $\lambda_{n}\left(L_{n}\right) \geqq a_{n} / n$. Define $G_{n-1}, a_{n-1}, b_{n-1}, \cdots, G_{1}, a_{1}, b_{1}$ similarly. By the hypothesis there is a real harmonic function $u$ on $D$ such that $\|u\| \leqq 2 M, u\left(z_{j}\right)=$ -2 if $z_{j} \varepsilon L_{n}, u\left(z_{j}\right)=2$ if $z_{j} \varepsilon L_{k}, k<n$. By (b), $u<-1$ on $L_{n}$ and $u>1$ on $L_{k}, k<n$. Letting $\lambda_{\zeta}$ be the harmonic measure for $\zeta \varepsilon L_{n}$ on $\partial G_{n-1}$, we have by the maximum principle

$$
-1>\lambda_{\zeta}\left(\bigcup_{k<n} L_{k}\right)-2 M\left(1-\lambda_{\zeta}\left(\bigcup_{k<n} L_{k}\right)\right)
$$

so that

$$
\lambda_{\zeta}\left(\bigcup_{k<n} L_{k}\right)<\frac{2 M-1}{2 M+1}=\alpha
$$

Hence

$$
a_{n-1} \leqq \alpha b_{n}+\left(a_{n}-b_{n}\right) \leqq \frac{(n-1)+\alpha}{n} .
$$

Repeating this gives

$$
a_{1} \leqq \prod_{k=1}^{n-1} \frac{k+\alpha}{k+1}<\epsilon
$$

provided $n$ is large enough. 
Map $D$ to $H$ by $z \mapsto e^{z}$. Then $L_{1}$ falls on a collection of radial slits in $0<x<e$, and by Hall's lemma (see [3] p. 208) these slits project angularly onto a set of small length. Mapping this projection back to $\bar{D}$, we see $\left|E_{n}\right|<1 / 2$ if $\epsilon$ is sufficiently small.

§3. Other interpolations. The corresponding problem for $L_{p}, 1 \leqq p<\infty$ does not require the construction and is thus much easier. Here one considers the mapping $T_{p}$ on $L^{p}[0,2 \pi]$ defined by the Poisson kernel

$$
T_{p} f\left(z_{j}\right)=\frac{1}{2 \pi} \int f\left(e^{i \theta}\right) \frac{1-\left|z_{j}\right|^{2}}{\left|e^{i \theta}-z_{j}\right|^{2}} d \theta .
$$

Now if $f \varepsilon H^{p}$, then

$$
\left(1-\left|z_{j}\right|\right)^{1 / p}\left|T_{p} f\left(z_{j}\right)\right| \rightarrow 0
$$

(see [3]), so it is natural to define an $H^{p}$ interpolating sequence as one for which $T_{p}\left(H^{p}\right)=L^{p}(\mu)$. The converse of the Carleson theorem used to show (3) implies (4) shows that (3) holds if $T_{p}: L^{p}[0,2 \pi] \rightarrow L^{p}(\mu)$ is bounded when $1<p<\infty$ or if $T_{1}$ is weak type $(1,1)$. Variants of Lemmas 1 and 2 then show that $(6)$ holds if $T_{p}\left(L^{p}\right)=L^{p}(\mu)$, and this means $T_{p}\left(H^{p}\right)=L^{p}(\mu)$.

Finally, since the interpolation problem is local, the theorem still holds on any finite open Riemann surface. We now show by example that it fails on an infinitely connected plane domain. Let $D=\Delta \backslash \bigcup_{j=1}^{\infty} \Delta_{i}$, where the $\Delta_{i}$ are pairwise disjoint open discs with centers on $x>0$ clustering at 0 . See [6] for information on such domains. The $\triangle_{i}$ can be chosen so that 0 is a regular point of $\partial D$ but so that the measure $d z / z$ on $\partial D$ has finite total variation. It follows that $\lim _{x \rightarrow 0-} f(x)$ exists for every bounded analytic function $f$ on $D$. However, we can choose inductively a sequence $x_{1}<x_{2}<\cdots$ with $\lim x_{n}=0$ such that if $\lambda_{n}$ is the harmonic measure for $x_{n} \varepsilon D$ on $\partial D$, there is a constant $M$ such that

$$
\sum\left|c_{j}\right| \leqq M\left\|\sum c_{j} \lambda_{j}\right\|
$$

for every sequence $\left\{c_{j}\right\}$. But (13) is the dual way of saying that $\left\{x_{j}\right\}$ is an interpolating sequence for the bounded harmonic functions on $D$. This example was first noticed by S. Fisher.

\section{ReFerences}

1. L. Carleson, An interpolation problem for analytic functions, Amer. J. Math. 80(1958), 921-932.

2. L. Carleson, The corona theorem, Proceedings of the 15th Scandinavian Congress, Oslo 1968. Lecture Notes in Mathematics, Vol. 118, Springer-Verlag.

3. P. L. Duren, Theory of $H^{p}$ spaces, Academic Press, New York, 1970.

4. K. Hofrman, Banach spaces of analytic functions, Prentice-Hall, Englewood Cliffs, New Jersey, 1962.

5. L. Hörmander, $L^{p}$ estimates for (pluri-) subharmonic functions, Math. Scand. 20(1967), $65-78$. 
6. L. Zalcman, Bounded analytic functions on infinitely connected domains, Trans. Amer. Math. Soc. 144(1969), 241-270.

Research partially supported by NSF Grant GP-11475.

University of California

Los Angeles

Date Communicated: February 15, 1971 